\title{
Computational Logic and Continuous Mathematics, Pure and Applied
}

\author{
Rob Arthan \\ Lemma 1 Ltd. \\ 2nd Floor, 31A Chain Street, Reading RG1 2HX, UK \\ and \\ QMUL, School of Electronic Engineering and Computer Science, \\ Queen Mary, University of London, London E1 4NS, UK \\ rda@lemma-one.com
}

Continuous problem domains are of ever-increasing importance in the application of computational logic to problems in systems engineering and to problems in mathematics and theoretical computer science. I will outline some recent work both "pure" and "applied" with issues for mechanized reasoning and computer algebra in mind.

For some years, I have been involved with tools used for formally specifying and verifying digital subsystems of avionics control systems. The models used in this work typically have discrete time and continuous data. These discrete models emerge only at the end of a chain of refinements starting from a purely continuous top-level model of the overall system. I will describe a strand of work on methods for dealing with linear continuous systems and discuss issues for mechanized reasoning that are highlighted by this methodological research.

I will sketch some joint work with Robert M. Solovay and John Harrison into decidability and undecidability for various theories of normed spaces and inner product spaces. These are theories that occur naturally both in engineering applications and in applying mechanized reasoning to pure mathematics. On the positive side, we have decision procedures for inner product spaces and for the some fragments of the theory of normed spaces. On the negative side, we can prove undecidability for the theory of normed spaces in general. However, there are still interesting open problem areas to investigate. It is noteworthy that one of the constructions used in the undecidability results provides an interesting challenge problem for mechanized proof and computer algebra. 